Z. klin. Chem. u. klin. Biochem.

10. J.g. 127.2, S. $121-126$

\title{
One-line-Betrieb von Digitalphotometer und Kleinrechner
}

\author{
Von W. KöRBER und W. RICK

\begin{abstract}
Kliniscb-chemiscbe Abteilung (Leiter: Prof. Dr. W. Rick) der I. Medizinischen Klinite (Dircktor: Prof. Dr. F. Grosse-Brockboff) der Liniversität Düsseldorf
\end{abstract}

(Eingegangen am 7. Oktober 1971)

Es werden Anwendungsmöglichkeiten einer Kombination aus dem Digitalphotometer Leitz DP digital und dem Mikrocomputer Olivetti P 203 anhand von zwei Beispielen beschrieben.

\author{
The on-line operation of a digital photometer and a desk computer
}

Two examples are presented for the combination of the digital photometer Leitz DP digital and the Olivctti P 203 computer.

Bei den meisten im klinischen Laboratorium verwendeten teil- oder vollmechanisierten Analysengeräten werden die photometrisch ermittelten Meßergebnisse in analoger Form ausgegeben. Die weitere Auswertung erfolgt durch Ablesung eines dem Analogwert entsprechenden digitalen Wertes, der in eine Konzentration oder eine Enzymaktivität umgerechnet wird. Jeder dieser Teilschritte ist mit mehr oder weniger großen Fehlern behaftet. Hier dürften einige der Gründe dafür liegen, daß die Präzision der Analysenergebnisse mit der Einführung mechanischer Systeme nicht in dem erwarteten Maße zugenommen hat (1). Einige der oben genannten Teilschritte des Arbeitsablaufes können einer EDV-Anlage übertragen werden. Bei den Vorbereitungen zur Anwendung solcher Anlagen im klinisch-chemischen Laboratorium stellt sich die Frage nach der Schnittstelle zwischen Meßgerät (meist Photometer) und EDV-Anlage; diese Frage wird in letzter Zeit einheitlich dahingehend beantwortet, daß die Schnittstelle hinter dem digital anzeigenden Photometer liegen sollte $(2-5)$. Dabei ist es zunächst unerheblich, ob am Ausgang des Photometers nur die Extinktion in digitaler Form vorliegt, oder ob im Meßgerät auch bereits Berechnungen von Konzentrationen vorgenommen werden.

Im folgenden soll eine Kombination zwischen einem digital anzeigenden Spektrallinienphotometer und einem Bürocomputer beschrieben werden, die im on-lineBetrieb arbeitet und sich praktisch bewährt hat. Die Anlage wurde so programmiert, daß sie in der Lage ist, aufgrund logischer Entscheidungen eine Aussage über die Qualität der Analysen zu machen und auf diese Weise die Zuverlässigkeit der klinischen Untersuchungen zu verbessern. Anhand einer Endpunktmessung (Gesamt-Bilirubin) und einer kontinuierlichen Messung (alkalische Phosphatase) soll die Leistungsfähigkeit des Systems gezeigt werden.

\section{Geräte}

\section{Photometer}

Als Meßgerät wird das Doppelstrahl-Photometer DP digital (Leitz, Wetzlar) verwendet. Das Gerät ist mit einer Wolframlampe und einer $\mathrm{Hg}$-Lampe ausgerüstet. Wegen der meßtechnischen Vorteilc, die eine monochromatische Strahlung bietet, verwenden wir nur die $\mathrm{Hg}$-Lampe als Strahlungsquelle, deren Linien bei 334, 366, 405, 436, 546 und $578 \mathrm{~nm}$ durch Filter isoliert werden. Alle Filter befinden sich in einer Revolverscheibe, die von der Frontseite des Gerätes bedient wird, so daß beim Wechsel des Filters der Küvettenraum nicht geöfnet zu werden braucht. Die Wellenlänge des jeweils benutzten Filters wird bei der Datenausgabe mitgedruckt. Uber eine Eingabeschaltereinheit kann zusätzlich das Datum, eine zweistellige Methodennummer und eine zweistellige Seriennummer ausgegeben werden.

Der Abgleich zwischen Verglcichs- und Meßstrahl erfolgt nach Druck auf eine entsprechende Taste und außerdem vollautomatisch nach jedcm Filterwechsel. Neben dem einfachen Küvettenhalter mit zwei Küvetten steht in Verbindung mit der Küvettenwechselautomatik ein temperierbarer Küvettenhalter für fünf Küvettenpaare zur Verfügung. Dabei dient das erste Küvettenpaar für den Abgleich, die weiteren vier Küvettenpaare sind für $\mathrm{Meß}$ - und Vergleichslösungen bestimmt. Neben Einzelmessungen können mit der Automatik für alle vier Küvettenpaare Meßzyklen von $20,30,60,120$ und 240 Sekunden Dauer durchgeführt werden. Der Zyklus wiederholt sich solange, bis er von Hand abgestellt wird; es kann jedoch auch ein fünfmaliger Durchlauf mit automatischer Abschaltung gewählt werden.

Der Meßwert kann als Transmission, Extinktion oder Konzentration vierziffrig und kommarichtig direkt am Gerät abgelesen und gleichzeitig im BCD-Code einem Rechner übermittelt werden.

Das Photometer ist weiter ausbaufähig mit Gitter-Monochromator und Zusätzen für fluorometrische Messungen sowie Remissionsmessungen. Es ist möglich, einen Kompensationsschreiber anzuschließen.

Rechner und Ausgabeeinheit

Als frei programmierbarer Rechner dient ein Olivetti-Bürocomputer P 203, bei dem die Ausgabe der Daten wahlweise über die eingebaute elcktrische Schreibmaschine oder über einen Streifendrucker erfolgen kann. Der Rechner verfügt über cinen magnetostriktiven Laufzeitspeicher, der in 10 Register aufgeteilt ist. Jedes Register besteht aus 32 Speicherstellen zu je 8 Bits 
(2 Register: Ausschließlich Befehlsspeicherung; 2 Register: Ausschlicßlich Zahlenspeichcrung; 3 Register: Zahlen-und/oder Befehlsspeicherung; 3 Operationsregistcr). In den Befehlsregistern können maximal 160 Befehle gespeichert werden. Es ist möglich, die als Zahlenspeicher dienenden Register zu unterteilen, so daß 10 Register zur Zahlenspeicherung zur Verfügung stehen.

Das Einlesen des Programms erfolgt durch Magnetkarten. Neue Programme können ohne weitere Hilfsmittel selbst entwickelt werden. Sie werden über die Rechnertastatur eingegeben und anschließend auf Magnetkarten festgehalten. Mit der eingebauten Schreibmaschine ist es möglich, über Programmbefehle numerische Daten formulargerecht auszudrucken.

Photometer und Rechner sind über ein Interface on-line verbunden. Zur Inbetriebnahme ist lediglich das benötigte Programm durch Magnetkarte einzugeben. $\mathrm{Da}$ dies nur Sekunden erfordert, kann innerhalb kürzester Zeit von einer Meßmethode auf die andere übergegangen werden. Die Gerätekombination wird in unserem Laboratorium deshalb vorzugsweise zur Auswertung von Meßreihen eingesetzt, für die sich die Anschaffung von teil- oder vollmechanisierten Analysengeräten nicht lohnt oder für die die Umrüstung vorhandener Systeme zu umständlich und zeitraubend wäre. Beide Geräte sind getrennt außerhalb der Routine für vielfältige Zwecke benutzbar. So werden unsere gesamten statistischen Berechnungen mit der Olivetti P 203 ausgeführt.

\section{Anwendungsbeispiele}

1. Bestimmung der Konzentration des GesamtBilirubins

Methodik

Zur Bestimmung der Bilirubinkonzentration im Serum dient die Methode von Jendrassik und Grof (6) im Mikrolitermaßstab. Die Serummenge pro Test beträgt $100 \mu \mathrm{l}$, das Endvolumen $1200 \mu \mathrm{l}$. Wegen der mit dem üblichen FeHLING II-Reagenz nicht selten auftretenden Trübung wird ein weniger konzentriertes Reagenz verwendet, das $0,4 \mathrm{M}$ Kalium-Natrium-Tartrat in $1 \mathrm{~N}$ Natronlauge enthält. Jede Probe wird zweifach analysiert.

\section{Programm}

Aus den beiden Extinktionsdifferenzen zwischen Serumleerwerten und Bestimmungsansätzen wird der Mittelwert errechnet. Bei einer mittleren Extinktionsdifferenz unter 0,200 ist eine Abweichung der Extinktionen vom Mittelwert bis maximal $5 \%$ erlaubt, bei einer mittleren Extinktionsdifferenz über 0,200 eine Abweichung bis zu maximal 2,5\%. Bei größeren Unterschieden wird anstelle des Ergebnisses 0.000 gedruckt. Werden die Extinktionsdifferenzen vom Rechner akzeptiert, so wird der Mittelwert mit dem Faktor 11,3 multipliziert und das Ergebnis, auf eine Stelle hinter dem Komma auf- $(\geqq 0,05)$ oder abgerundet $(<0,05)$, in $\mathrm{mg} / 100 \mathrm{ml}$ angegeben. Bei Extinktionsdifferenzen über 0,800 sind die Bedingungen des LAMBERT-BeER'schen Gesetzes nicht mehr gegeben. Die Probe muß verdünnt angesetzt werden, es wird anstelle des Ergebnisses 9.999 gedruckt. Die Programmbefehle sind im Anhang wiedergegeben.

Ausdruck

Am Kopf des Formulares (siehe Abb. 1) einmal Dâtum und Filter, dann zeilenweise von links nach rechts
Extinktionsdifferenz 1, Extinktionsdifferenz 2, Mittel der Extinktionsdifferenzen, Ergebnis in $\mathrm{mg} / 100 \mathrm{ml} \mathrm{bzw.}$ 0.000 bzw. 9.999.

\section{Arbeitsablauf \\ Vorbereitungen am Pbotometer}

Photometer 20 Min. einbrennen lassen.

Filter $578 \mathrm{~nm}$ in den 'Strahlengang bringen, Datum einstellen, Küvetten mit bidest: ' Wasser füllen und automatischen Abgleich betätigen.

\section{Vorbereitungen am Recbner}

Das Formular, in dem die Patientendaten eingetragen sind, einspannen. Interface einschalten, Netzschalter von Rechner und Schreibmaschine einschalten, Löschtaste drücken, Magnetkarte durchlaufen lassen, Taste V drücken.

\section{Messung}

In die Küvetten ersten Leerwert und ersten Meßansatz einfüllen. Drucktaste betätigen. Zweiten Leerwert und zweiten Meßansatż einfüllen. Drucktaste betätigen. Während der folgenden 9 Sek. werden Berechnungen, Ausdruck und Zeilensprung ausgeführt. In dieser Zeit ist die elektronische Funktion des Photometers gesperrt, so $\mathrm{da} \beta$ eine Übermittlung von Daten zum Rechner nicht möglich ist. Anschließend Einfüllen der Meßlösungen und Übergabe $\operatorname{der}$ Meßwerte an den Rechner wie beschrieben fortsetzen.

Am Ende der Meßreihe Taste W des Rechners drücken; die Anzahl der Analysen wird auf dem Protokoll ausgedruckt (siehe Abb. 1).

\section{Bestimmung der Aktivität der alkalischen Phosphatase \\ Methodik}

Kontinuierliche Messung der Enzymaktivität mit $p$ Nitrophenylphosphat in optimaler Konzentration als Substrat (7). Messung in Normalküvetten, $1 \mathrm{~cm}$ Schichtdicke, Filter $405 \mathrm{~nm}$, Meßtemperatur 25 4 Proben werden fünfmal im Abstand von jeweils. einer Minute gemessen.

\section{Programm}

Das Programm besteht aus zwei Teilen (vgl. Anhang), die getrennt auf zwei Magnetkarten festgehalten sind.

\section{Programmteil I}

Übernahme und Ausdruck der Extinktionen, Errechnung, Ausdruck und Speicherung der Extinktionsdifferenzen aller vier Proben für fünf Durchläufe.

\section{Programmteil II}

Von jeder der vier Proben wird nacheinander der Mittelwert der vier Extinktionsdifferenzen errechnet und jede einzelne Extinktionsdifferenz darauf untersucht, ob sie um mehr als 10\% vom Mittelwert abweicht. Ist dies der Fall, wird die betreffende Extinktionsdifferenz eliminiert. Weichen zwei Extinktionsdifferenzen um 


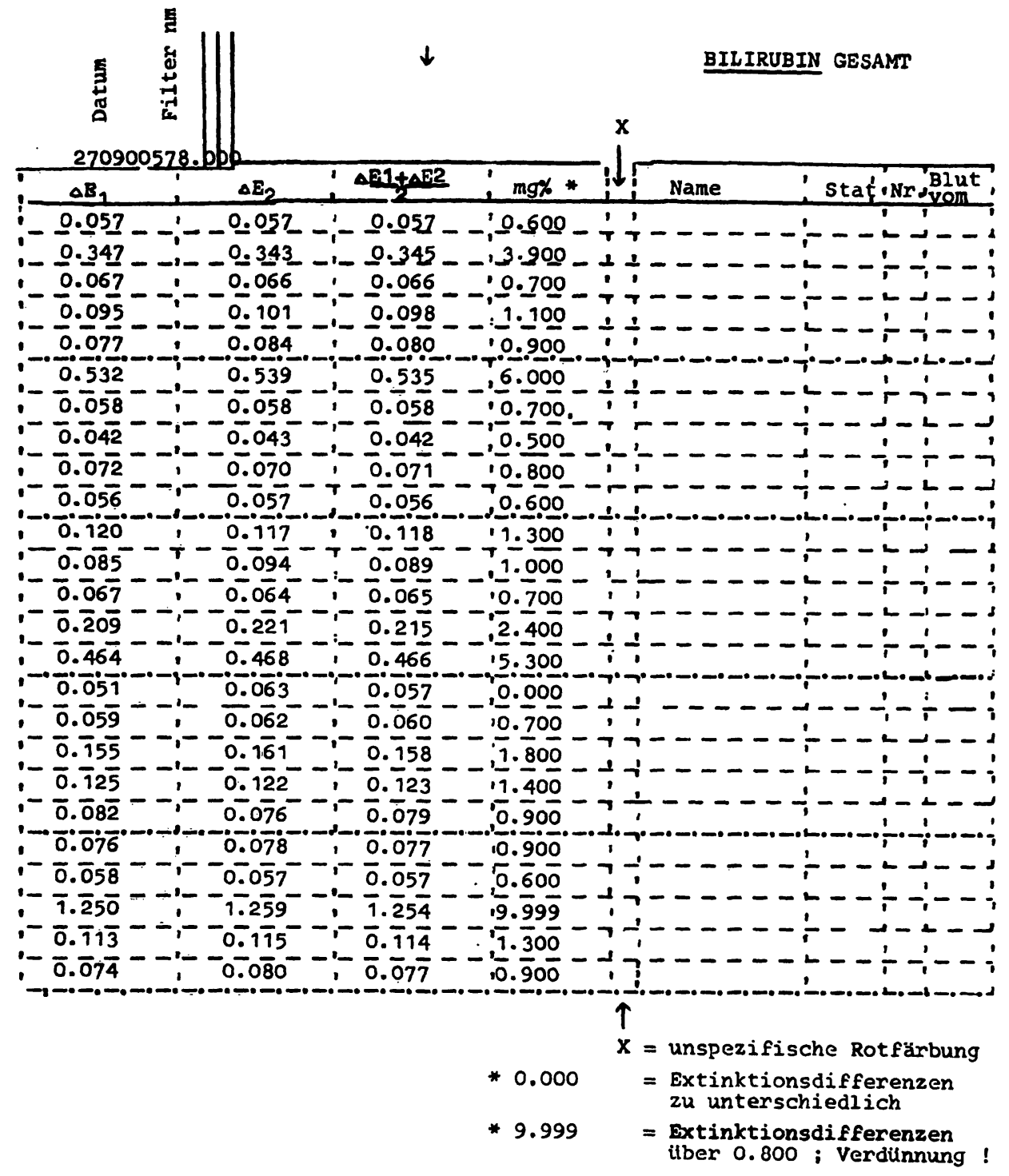

25.000

Anżạhl

Untersuchung durchgefuhrt von :

Befunde geschrieben von

:- - - - - -

Abb. 1

Bilirubinbestimmung. Ausdruck der Ergebnisse

mehr als $10 \%$ vom Mittelwert ab, so wird keine Enzymaktivität errechnet. Werden die Extinktionsdifferenzen akzeptiert, so wird der Mittelwert mit dem Berechnungsfaktor 5460 multipliziert und das Ergebnis in $\mathrm{mU} / \mathrm{ml}$ ausgedruckt.

\section{Ausdruck}

$\mathrm{Zu}$ Beginn eines Meßzyklus werden einmal Datum und Filter, dann zeilenweise von links nach rechts die jeweils zusammengehörenden Extinktionen und Extinktionsdifferenzen der Proben 1 bis 4 ausgedruckt (siehe Abb. 2). Unter der jeweiligen Zahlenkolonne erscheint das Ergebnis in $\mathrm{mU} / \mathrm{ml}$. Werden die Meßwerte verworfen, so wird nichts gedruckt, die Schreibmaschine rückt lediglich bis zum nächster Tabulatorstand weiter.

\section{Arbeitsablauf}

\section{Vorbereitungen am Pbotometer}

Photometer 20 Min. einbrennen lassen, Filter $405 \mathrm{~nm}$ vorlegen. An der Eingabeschaltereinheit das Datum und an der Küvettenautomatik Zykluszeit (60 Sek.) einstellen, Temperatur des Thermostaten für den Küvettenhalter $\left(25^{\circ}\right)$ kontrollieren, vier Küvetten mit $2,0 \mathrm{ml} p$-Nitrophenjlphosphat/Puffer-Gemisch, auf $25^{\circ}$ temperiert, füllen.

\section{Vorbereitungen am Recbner}

Das Formular, auf dem Datum und Patient eingetragen sind, einspannen, Interface einschalten, Netzschalter von Rechner und Schreibmaschine einschalten, Löschtaste drücken, Magnetkarte mit Programmteil I durchlaufen lassen. Taste $\mathrm{V}$ drücken. 


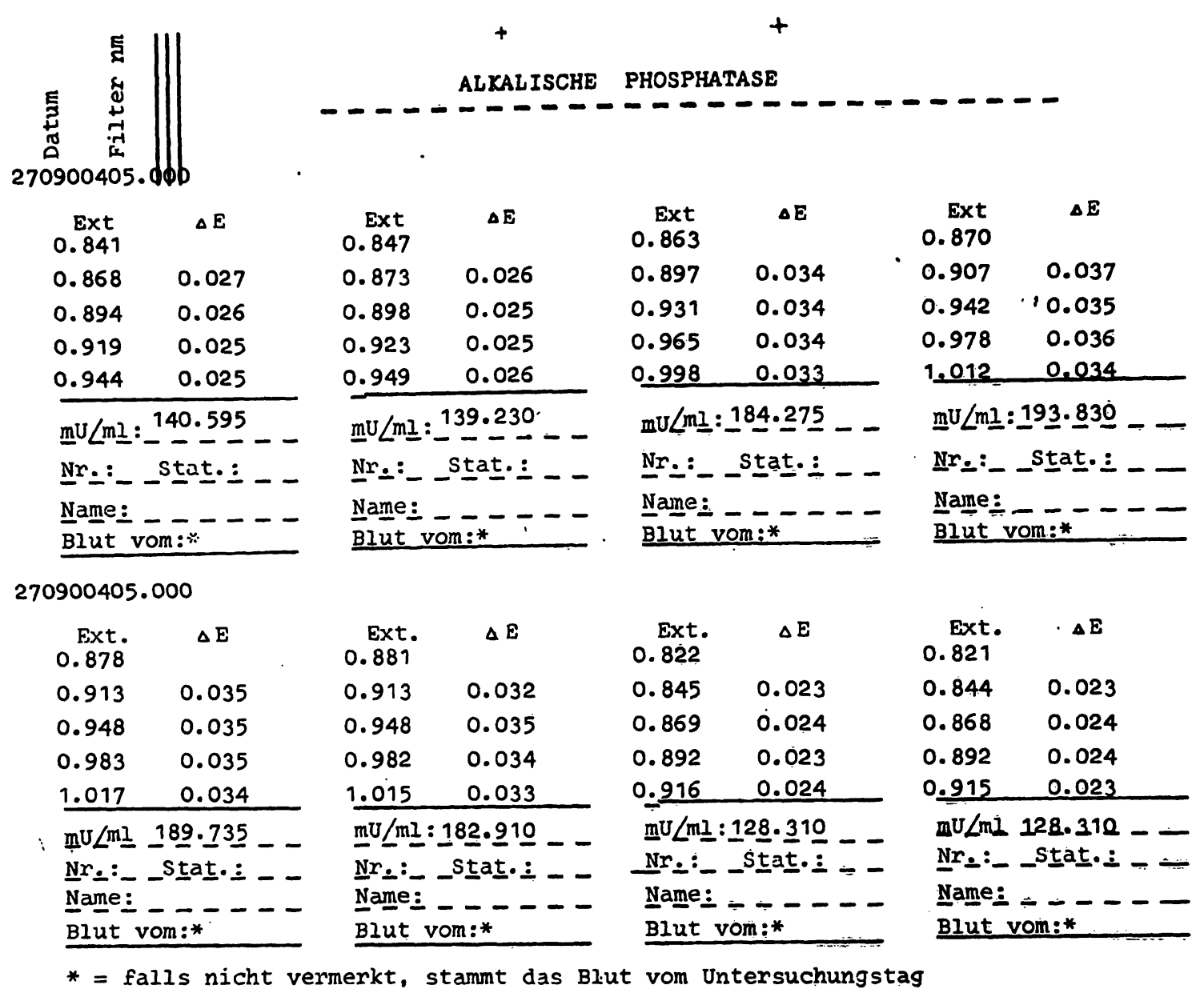

Abb. 2

Bestimmung der Aktivität der alkalischen Phosphatase. Ausdruck der Ergebnisse.

\section{Messung}

$20 \mu l$ Serum in die vorbereiteten Küvetten pipettieren, mit Plastikspatel gut mischen.

Starttaste der Küvettenautomatik drücken. Der Zyklus läuft $5 \mathrm{mal}$ automatisch hintereinander ab.

\section{Auswertung}

Nach Ablauf des Zyklus Magnetkarte mit Programmteil II durchlaufen lassen und Taste $\mathrm{V}$ des Rechners drücken. Der Ausdruck erfolgt wie oben beschrieben (siehe Abb. 2).

Während des Rechenvorganges kann die nächste Serie von Meßansätzen vorbereitet werden. Es ist vorteilhaft, einen zweiten Küvettenhalter anzuschaffen, der bereits während des vorhergehenden Durchlaufs beschickt wird. Mit fortlaufendem Austausch der temperierten Küvettenhalter durch einfaches Abheben von der Trägerplatte läßt sich so eine hohe Arbeitsgeschwindigkeit erzielen.

\section{Diskussion}

Im klinisch-chemischen Laboratorium werden bisher fast ausschließlich Photometer verwendet, bei denen die Meßergebnisse in analoger Form abgelesen bzw. registriert werden. Sollen die Analogwerte in Digitalwerte umgeformt werden, so sind Zusatzgeräte erforderlich, die nach verschiedenen Prinzipien arbeiten
(8) und meist auch die Umrechnung von Transmissionen in Extinktionen bzw. Konzentrationen ermöglichen (9). Bei dem Doppelstrahlphotometer DP digital wird nach einem Meßprinzip, auf das in diesem Zusammenhang nicht weiter eingegangen werden soll, das Meßsignal nach Verstärkung direkt in die digitalisierte Extinktion umgewandelt, so $\mathrm{da} /$ die Darstellung der Transmission in analoger Form und die weiteren Umrechnungen entfallen.

Elektronische Kleinrechner sind im Laboratorium schon weit verbreitet. Bisher mußten jedoch die zur Verarbeitung vorgesehenen Meßwerte manuell eingegeben werden (10), so daß der Rationalisierungseffekt bei dieser Art der Anwendung gering sein dürfte. Voraussetzung für den on-line-Betrieb ist die Möglichkeit zur direkten Eingabe, die bei Kleinrechnern erst seit kurzem vorhanden ist.

Bekanntlich ist jede Messung mit unvermeidlichen Fehlern behaftet. Die Interpretation der am Untersuchungsmaterial eines Probanden ermittelten Analysenergebnisse beruht auf dem Vergleich mit dem für die betreffende Methode gültigen Normbereich (Transversal-Beurteilung) und mit früheren, am gleichen Probanden erhobenen Befunden (Longitudinal-Beurteilung). Ein solcher Vergleich ist jedoch nur möglich, wenn das verwendete Analysensystem stationär, d. h. unter Kontrolle ist; dies ist durch die Maßnahmen der 
mit dem soeben erschienenen

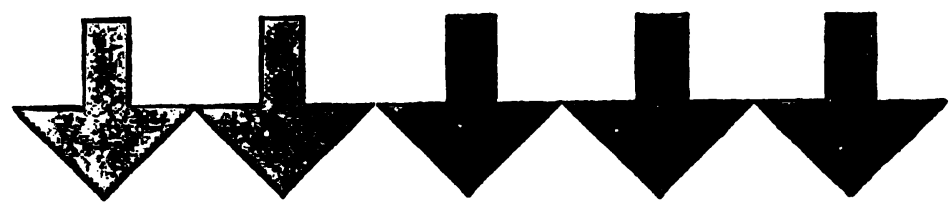

\section{Handbuch der}

\section{Prasisrationalisierung}

von Dr. med. HANS-JÜRGEN FRANK-SCHMIDT

und Professor Dr. Dr. EMIL HEINZ GRAUL

564 Seiten mit 406 Fotos, Zeichnungen und Vordrucken sowie zahlreichen Tabellen, Formularen und Vertragswerken, Plastikeinband, DM 110,-

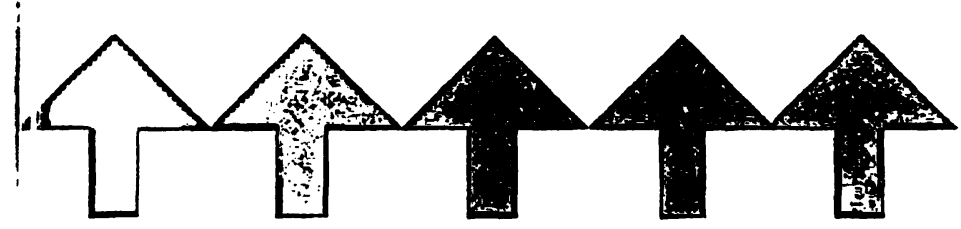

Da kein Arzt alle organisatorischen und technischen Möglichkeiten überschauen und erst recht nicht beurteilen kann und nicht erst teuere und bittere Erfahungen selbst machen will, wird er sich des Rates von Standeskollegen bedienen, die das Angebot kennen 'und eine Auswahl treffen. Die Auswahl durch die Ver'fasser erfolgte ohne kommerzielle Interessen.

Der hohe Gebrauchswert des Handbuches liegt darin, $\mathrm{da} B$ es optimale Problemlösungen beispielhaft darstellt. Das gesamte Angebot an Dienstleistungen, Organisationsformen und Geräten wird erschlossen.

Aus dem Inhalt:

Vor der Planung der Niederlassung: Steuerplanung

Die Planung und Finanzierung der Praxis

Leasing und was der Arzt davon wissen muß

Die Mieten einer Praxis

Der rationelle Grundriß für einen Praxisneubau

Allgemeine Baumaßnahmen für Neubauten, Umbauten,

Anbauten und Praxiserweiterung

Gedanken zur Innenarchitektur einer Praxis:

Mobiliar, Büromittel, Labor, u. ähni.

Die Ankündigung der Niederlassung

Die Finanzplanung nach der Niederlassung u. v. a.

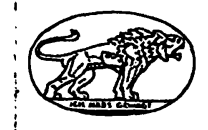

J. F. LEHMANNS VERLAG MUNCHEN

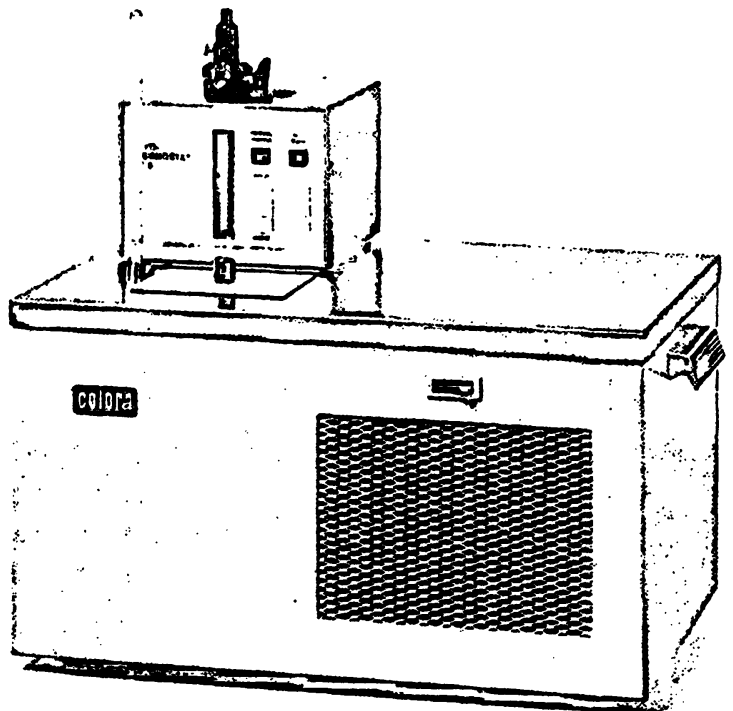

\section{Colora-} Kryo-Thermostat WK 5

Ideales Tischgerät für Temperierungen im Bad und Umwälzverfahren.

Arbeitsbereich von $-15^{\circ} \mathrm{C}$ bis $+150^{\circ} \mathrm{C}$. Durch eingebautes Kälteaggregat unabhängig von äußerer Kühlung und dadurch jederzeit im gesamten Bereich verwendbar. Funkentstörtes Elektronikrelais. Temperaturkonstanz $\pm 0,02^{\circ} \mathrm{C}$. Hochwertige Ausführung aus rostfreiem Stahl. Lagerfreie Umwälzpumpe hoher Leistung. Ein zuverlässiges Gerät.

\section{Colora Messtechnik GmbH} 7073 Lorch/Württ., Postfach 5 T (07172) 6041, FS 07-248886

Technische Büros (Verkauf und Kundendienst): 1000 Berlin 30, Kurfürstenstraße 84, T 2615200 2000 Hamburg 19, Osterstraße 63, T 491 1034, FS 02-12 947 3000 Hannover, An der Tiefenriede 45, T 884500 4000 Düsseldorf, Kronprinzenstr. 62, T 3201 64, FS 08-587 253 6000 Frankfurt a.M., Röderbergweg 4-6,T446031, FS 04-11216 8000 München 19, Dachauer Straße 175, T 193858

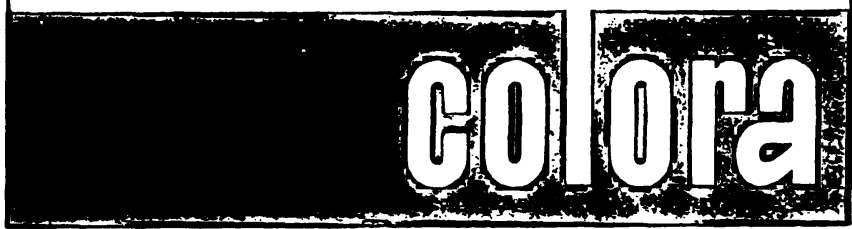




\section{Zw圈eruhigung Dhries Gewissens:} HYLAND Richtigkeits-und Präzisionskontrollen:

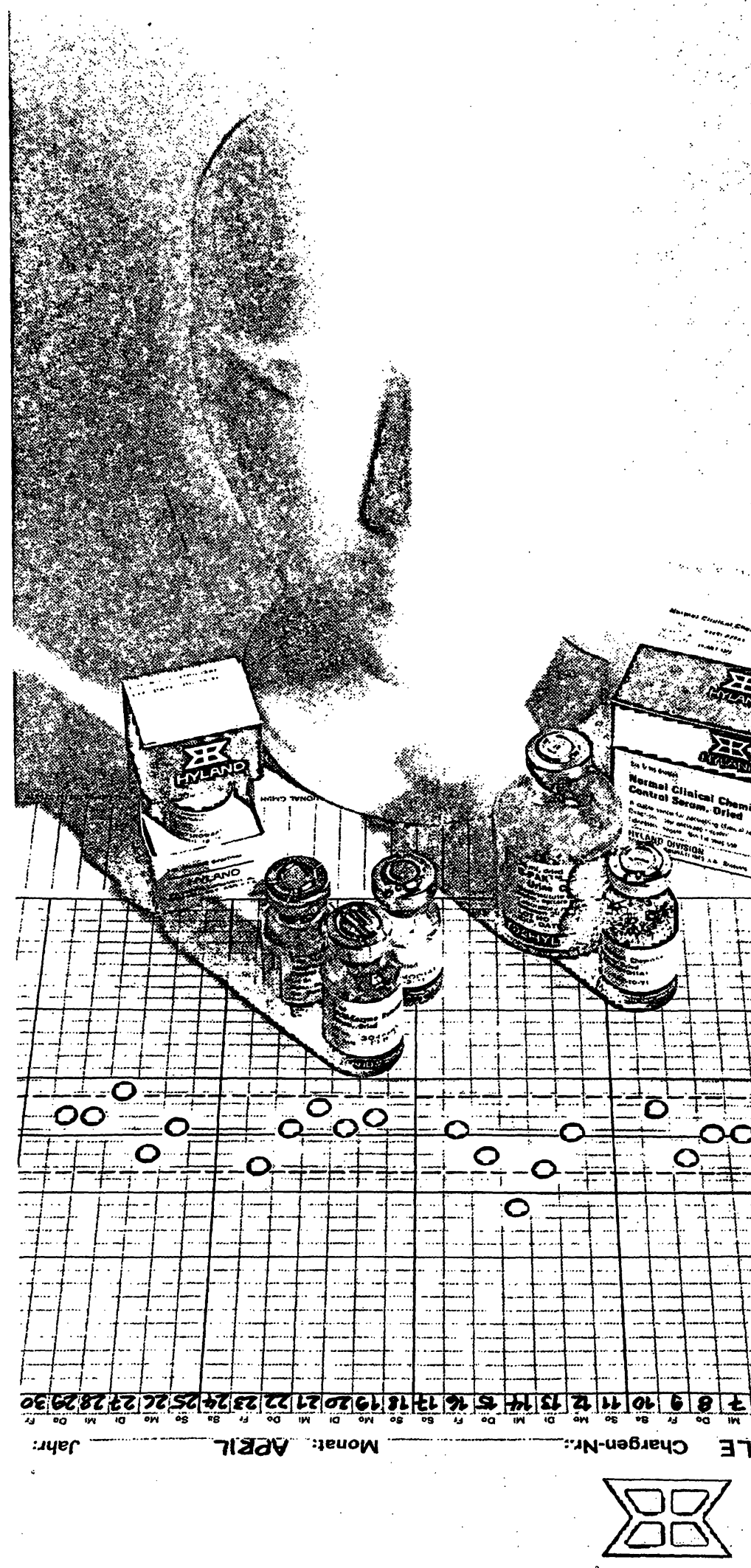

Präzisionskontrollen

Humanseren, Normal - Pathologisch Humanserum ${ }_{n} \mathrm{Q}$ Pak" für Analyseautomaten

\section{Richtigkeitskontrollen}

Humanseren, Normal"- Pathologisch"Spezial

Humanserum ${ }_{n} \mathrm{Q} P a k^{n}$ für Analyseautomaten Multi-Enzym-Referenzserum

Bilirubinkontrolle mit erhöhten Werten Cholesterinkontrolle mit erhöhten Werten

Kontrollurine. Kontroll-Liquor HB-Referenzstandard und-Kontrolle

"Mit Europawerten

Mit HYLAND-Kontrollreagenzien können Sie mehr als 70 verschiedene Bestimmungen überprüfen.

Verlangen Sie bitte unsere Informationsunterlagen. 
statistischen Qualitätskontrolle nachzuweisen $(1,11,12)$. Darïber hinaus sollte es das Ziel der klinisch-chemischen Analytik sein, zum Analysenergebnis auch einen Vertrauensbereich anzugeben, in dem die tatsächliche Konzentration des untersuchten Bestandteils liegt (13). Dieser Vertrauensbereich läßt sich nach Wahl einer geeigneten statistischen Sicherheit $\mathrm{P}$ mit Hilfe der Präzision in der Serie ermitteln; die Präzision in der Serie kann nur aus den Ergebnissen von Mehrfachanalysen experimentell bestimmt werden (11). Auf die notwendigen Rechenverfahren kann hier nicht näher eingegangen werden (siehe dazu 1. c. (11, 14-16)). Weiterhin hat die Ausführung von Doppelanalysen den Vorteil, daß zahlreiche grobe Fehler leicht gefunden werden können. Außerdem kann nach WhIтBY, MrTCHELl und Moss (1) eine verminderte Präzision eines Verfahrens anhand der Ergebnisse von Zweifachbestimmungen auch dann erkannt werden, wenn die Analyse der üblichen Kontrollproben keinen Anhalt für eine Herabsetzung der Präzision ergeben hat. Aus diesen Gründen werden in unserem Laboratorium alle Untersuchungen als Doppelbestimmungen angeführt. Werden mit der beschriebenen Gerätekombination
Konzentrationen von bestimmten Bestandteilen im Serum u. a. gemessen (,Endpunktverfahren"), so trifft der Rechner aufgrund des Programms durch Vergleich der beiden Extinktionen eine Entscheidung darüber, ob ein Ergebnis ausgegeben werden darf oder nicht. Die Speicherkapazität des Kleincomputers reicht jedoch nicht aus, solche Berechnungen auch für die Enzymaktivitätsbestimmungen vorzunehmen. Hierbei werden die Meßwerte für einen Test darauf untersucht, ob sie eine geradlinige Extinktionszunahme ergeben. Ist dies. innerhalb der erlaubten Fehlerbreite nicht der Fall, so. wird keine Enzymaktivität berechnet. In modifizierter Form kann das Programm auch für NADH-abhängige Bestimmungsmethoden verwendet werden. Bei den Verfahren zur Messung von Enzymaktivitäten ist der Vergleich der beiden, an einer Probe gemessenen Aktivitäten anschließend getiennt vorzunehmen.

Abschließend ist darauf hinzuweisen, $\mathrm{da} \beta$ bei der hier beschriebenen Arbeitsweise Ablese-, Schreib- und Rechenfehler weitgehend entfallen. Der Ausdruck des verwendeten Filters ergibt eine wichtige zusätzliche Kontrolle. Die protokollgerechte Ausgabe der Ergebnisse vereinfacht den Arbeitsablauf.

\section{Anhang}

Programmbefehle für die Bestimmung von Gesamt-Bilirubin. Die Befehle sind spaltenweise von oben nach unten zu lesen
Programmbefehle für die Bestimmung der alkalischen Phosphatase. Die Befehle sind spaltenweise von oben nach unten zu lesen Programmteil I:

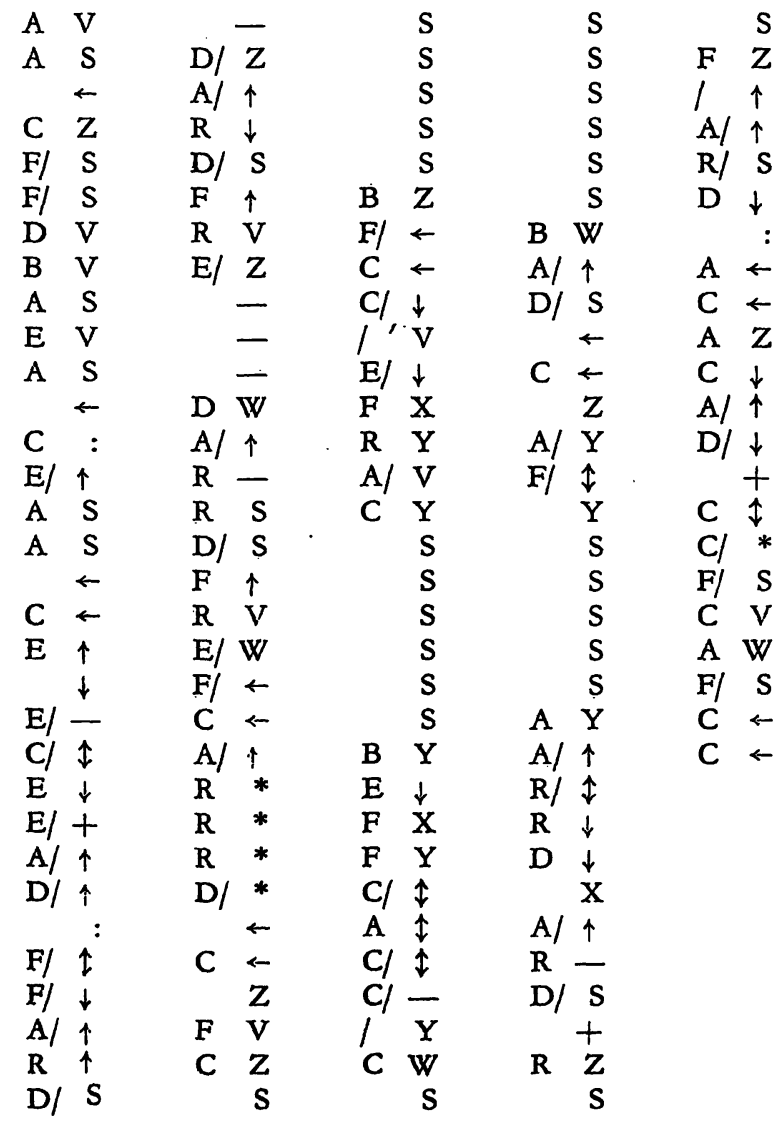

\begin{tabular}{|c|c|c|}
\hline A & $\mathrm{V}$ & \\
\hline $\mathrm{F} /$ & S & $D$ \\
\hline $\mathrm{F} /$ & S & D \\
\hline A/ & $\uparrow$ & $\bar{A}$ \\
\hline $\mathrm{D} /$ & $\downarrow$ & A \\
\hline D/ & $\uparrow$ & C \\
\hline$A^{\prime}$ & $\mathrm{S}$ & $\mathrm{D} /$ \\
\hline & $\leftarrow$ & B \\
\hline C & $\mathbf{Z}$ & B \\
\hline $\mathrm{F} /$ & $\mathrm{S}$ & A \\
\hline F/ & S & \\
\hline$A^{\prime}$ & $\mathrm{S}$ & C \\
\hline & $\leftarrow$ & \\
\hline$C$ & $\leftarrow$ & $\mathrm{E}$ \\
\hline$D$ & $\uparrow$ & $\mathrm{E} /$ \\
\hline A & $\mathrm{s}$ & A \\
\hline & $\leftarrow$ & A \\
\hline C/ & $\uparrow$ & C \\
\hline E/ & $\uparrow$ & $\mathrm{D} /$ \\
\hline$A^{\prime}$ & $\mathrm{S}$ & B/ \\
\hline & $\leftarrow$ & B) \\
\hline C/ & $\uparrow$ & A \\
\hline $\mathrm{E}$ & $\uparrow$ & \\
\hline A & $\mathrm{S}$ & C \\
\hline & $\leftarrow$ & \\
\hline C/ & $\uparrow$ & $E$ \\
\hline F/ & $\uparrow$ & $\mathrm{E}$ \\
\hline F/ & S & A \\
\hline$F$ & $\mathrm{~V}$ & $\mathrm{~A}$ \\
\hline A & $\mathbf{S}$ & C \\
\hline & $\leftarrow$ & D/ \\
\hline C & $\leftarrow$ & $\mathbf{R}$ \\
\hline
\end{tabular}

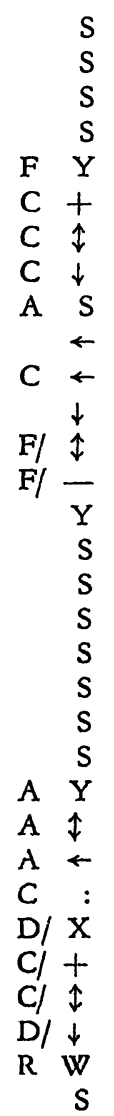

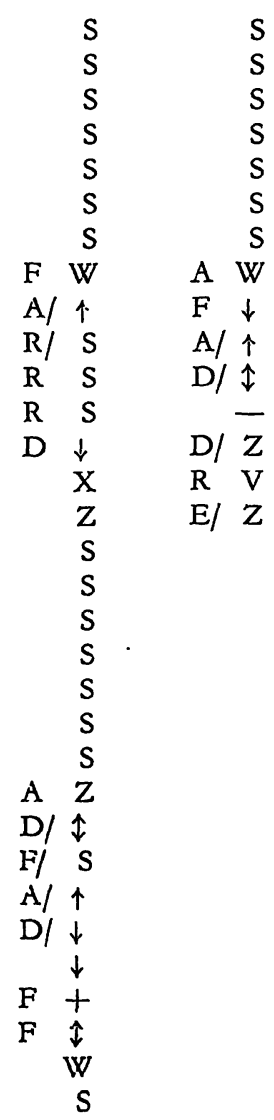


Programmteil II:

\begin{tabular}{|c|c|c|c|c|c|c|c|c|}
\hline & $\mathrm{S}$ & $\mathrm{F} /$ & $\downarrow$ & & $S$ & & $\mathrm{~S}$ & \\
\hline A/ & $\mathrm{V}$ & $E$ & $\uparrow$ & & $\mathrm{S}$ & & $\mathrm{S}$ & \\
\hline A) & $\uparrow$ & F/ & $\uparrow$ & & $\mathrm{S}$ & & $\mathrm{S}$ & \\
\hline $\mathrm{R} /$ & $S$ & A & $\uparrow$ & & $\mathrm{S}$ & & $S$ & \\
\hline $\mathbf{R}$ & $S$ & D & W & & $\mathrm{S}$ & & $\mathrm{S}$ & \\
\hline $\mathbf{R}$ & $S$ & $\mathbf{R}$ & V & F & W & F & $Z$ & A \\
\hline $\mathrm{D}$ & $\downarrow$ & $\mathrm{E}$ & W & I & W & & $Y$ & B \\
\hline D/ & $\uparrow$ & F & $\downarrow$ & $\mathrm{E}$ & $*$ & A/ & $Y$ & A \\
\hline A) & $\uparrow$ & $\mathrm{E}$ & $\mathfrak{\jmath}$ & A/ & $\uparrow$ & A/ & $\uparrow$ & B \\
\hline D/ & + & $F$ & $\uparrow$ & E/ & $\downarrow$ & R/ & $S$ & 1 \\
\hline$D$ & $\uparrow$ & A & $\uparrow$ & & $\downarrow$ & $\mathrm{R}$ & $\mathrm{X}$ & B/ \\
\hline 1 & $\uparrow$ & D & $Y$ & D & + & $\mathrm{R}$ & + & B/ \\
\hline$E$ & $\uparrow$ & $\mathrm{R}$ & $\mathrm{V}$ & D & $\uparrow$ & D & - & 1 \\
\hline D/ & : & $\mathrm{E}$ & $\mathrm{Y}$ & A/ & W & & $\downarrow$ & C \\
\hline 1 & $\uparrow$ & B & $\downarrow$ & & W & $\mathrm{R}$ & $Y$ & \\
\hline $\mathrm{F} /$ & 个 & $\mathrm{E}$ & $\uparrow$ & & $S$ & & $S$ & \\
\hline$D /$ & : & B & $\uparrow$ & & $S$ & & $S$ & \\
\hline I & $\uparrow$ & A & $\uparrow$ & & $S$ & & $S$ & \\
\hline$F$ & $\uparrow$ & D & Z & & $S$ & & $S$ & \\
\hline$D /$ & : & $\mathbf{R}$ & $\mathrm{V}$ & & S & & $S$ & \\
\hline 1 & $\uparrow$ & $\mathrm{F}$ & $\mathrm{V}$ & A & W & & $\mathrm{S}$ & \\
\hline B & $\uparrow$ & $\mathrm{E}$ & $\downarrow$ & $\mathbf{R}$ & $\uparrow$ & $\mathrm{F}$ & $Y$ & \\
\hline B & $\downarrow$ & $\mathrm{E} /$ & $1-$ & $E$ & Z & D & : & $\mathrm{A}$ \\
\hline $\mathrm{E}$ & + & A & $\uparrow$ & A/ & $\uparrow$ & B & $\uparrow$ & C \\
\hline $\mathrm{F}$ & + & D/ & $1 \uparrow$ & E/ & $\uparrow$ & $\mathrm{E}$ & + & I \\
\hline $\mathrm{F} /$ & + & E/ & $\downarrow$ & & $\downarrow$ & $\mathrm{F}$ & + & Cl \\
\hline D & : & A) & $1 \uparrow$ & D & + & $\mathrm{F} /$ & + & C/ \\
\hline E/ & $\uparrow$ & $\mathrm{R} /$ & s & 1 & $Y$ & B & $\mathrm{X}$ & 1 \\
\hline A & $\uparrow$ & D & $\downarrow$ & 1 & $\leftarrow$ & A & $\leftarrow$ & $\mathrm{F} /$ \\
\hline $\mathrm{D}$ & V & $\mathrm{R} /$ & $\mathrm{x}$ & C/ & $\uparrow$ & Cl & $\uparrow$ & $\mathrm{F} /$ \\
\hline $\mathbf{R}$ & V & D) & $1-$ & $\mathrm{R}$ & $\mathbf{Z}$ & & $\mathrm{Y}$ & $\mathrm{F} /$ \\
\hline$E$ & $\mathrm{~V}$ & $\mathbf{R}$ & W & & $S$ & & S & \\
\hline
\end{tabular}

\section{Literatur}

1. Whirby, L. G., F. L. Mrrchell und D. W. Moss, Adv. Clin. Chem. 10, 65 (1967). - 2. NeTHELER, H., Diskussionsbemerkung, Eppendorf-Diskussionskreis, Hamburg 1970. - 3. FAHR, E., Diskussionsbemerkung, Eppendorf-Diskussionskreis, Hamburg 1970. - 4. Brerens de HAAN, J., Vortr. Tagung schweiz. Vereinigung klin. Chem., Bașel 1971. - 5. HoLx, H. W., Vortr. Tagung schweiz. Vereinigung klin. Chem., Basel 1971. - 6. Jendrassik, L. und P. GroF, Biochem. Z. 297, 81 (1938). 7. Hausamen, T.-U., W. Rick, W. Gross und R. Helger, Clin. Chim. Acta, Amsterdam 15, 241 (1967). - 8. FärBer, G., dièse Z. 7, 214 (1969). - 9. Borner, K. und E. KLEIN, diese Z. 7, 185 (1969). - 10. Borner, K., diese Z. 6, 269 (1968). - 11. BürTNer, H., E. Hansert und D. Stamm, Auswertung, Kontrolle und Beurteilung von Mèßergebnissen, in Bergmeyer, H. U. (Hrsg.), Methoden der enzymatischen Analyse, 2. Aufl., Verlag Chemie, Weinheim (1970), Seite 282. - 12. Richtlinien der Bundesärztekammer zur Durchführung der statistischen Qualitätskontrolle und von Ringversuchen im Bereich der Heilkunde. Dtsch. Åtzteblatt 68, 2228 (1971). - 13. StAmM, D., Vortrag beim MerckSymposium „Auftrag der Klinik an das klinisch-chemische Laboratorium", Wiesbaden 1970 (im Druck). - 14. DoERFFEx, K., Beurteilung von Analysenverfahren und Ergebnissen, SpringerVerlag, Berlin-Heidelberg-New York (1965), Seite 33. - 15. Fucrs, G., Mathematik für Mediziner und Biologen. SpringerVerlag, Berlin-Heidelberg-New York (1969), Seite 159. - 16. DIN 1319, Blatt 3, Grundbegriffe der Meßtechnik, Begriffe für die Fehler beim Messen, Abs. 4. 3. Dtsch. Normenausschuß, Berlin (1968).

Prof. Dr. W. Rick 4000 Düsseldorf Moorenstr. 5 